\title{
Time-Varying Respiratory Pattern Characterization in Chronic Heart Failure Patients and Healthy Subjects
}

\author{
Ainara Garde, Student Member, IEEE, Beatriz F. Giraldo, Member, IEEE, Raimon Jané, Member, IEEE \\ and Leif Sörnmo, Senior Member, IEEE
}

\begin{abstract}
Patients with chronic heart failure (CHF) with periodic breathing (PB) and Cheyne-Stokes respiration (CSR) tend to exhibit higher mortality and poor prognosis. This study proposes the characterization of respiratory patterns in CHF patients and healthy subjects using the envelope of the respiratory flow signal, and autoregressive (AR) time-frequency analysis. In time-varying respiratory patterns, the statistical distribution of the AR coefficients, pole locations, and the spectral parameters that characterize the discriminant band are evaluated to identify typical breathing patterns. In order to evaluate the accuracy of this characterization, a feature selection process followed by linear discriminant analysis is applied. $26 \mathrm{CHF}$ patients (8 patients with $\mathrm{PB}$ pattern and 18 with non-periodic breathing pattern (nPB)) are studied. The results show an accuracy of $83.9 \%$ with the mean of the main pole magnitude and the mean of the total power, when classifying $\mathrm{CHF}$ patients versus healthy subjects, and $83.3 \%$ for nPB versus healthy subjects. The best result when classifying CHF patients into $P B$ and $n P B$ was an accuracy of $88.9 \%$, using the coefficient of variation of the first AR coefficient and the mean of the total power.
\end{abstract}

\section{INTRODUCTION}

Chronic heart failure (CHF) is often related to breathing abnormalities such as various forms of oscillatory breathing patterns characterized by rises and falls in ventilation. Periodic breathing $(\mathrm{PB})$ patterns can be classified into ventilation with apnea, commonly known as Cheyne-Stokes respiration (CSR), and ventilation without apnea [1], [2]. Some studies report a $70 \%$ PB prevalence in patients with CHF [3]. PB patterns and CSR predict poor prognosis and have been associated with higher mortality in patients with CHF [4], [5]. Different clinical studies have addressed the problem of defining physiological parameters which characterize such respiratory patterns [6], [7]. The patterns are also influenced by wakefulness or sleep, posture, and physiological and mental activity.

The origin of the PB pattern is still being debated among researchers. The respiratory modulation frequency appears to be essential in the understanding of periodic and non-

Manuscript received April 20, 2009. This work was supported in part by Ministerio de Ciencia e Innovación under grants TEC2007-68076-C02-01 and TEC2007-63637 from the Spanish Government.

A. Garde, B.F. Giraldo and R. Jané are with Dept. of ESAII, Universitat Politècnica de Catalunya (UPC), Institut de Bioenginyeria de Catalunya (IBEC) and CIBER de Bioingeniería, Biomateriales y Nanomedicina (CIBER-BBN). c/. Pau Gargallo, 5, 08028, Barcelona, Spain. (fax: +34 93401 7045) (e-mail: ainara.garde@upc.edu, beatriz.giraldo@upc.edu, raimon.jane@upc.edu).

L. Sörnmo is with the Department of Electrical and Information Technology and Center of Integrative Electrocardiology (CIEL), Lund University, Lund SE-22100, Sweden (e-mail: leif.sornmo@eit.lth.se). periodic breathing patterns in CHF patients. Thus, the envelope of the respiratory flow signal is studied in-depth. Our first studies characterized the relevant frequency band determined by the modulation frequency peak extracted from the respiratory flow envelope signal's power spectrum [8], [9]. Normal breathing frequency ranges from 12 to 20 breaths per minute (i.e., $0.20-0.33 \mathrm{~Hz}$ ), whereas the PB pattern is characterized by cycle lengths between 25 and 100 s (i.e., $0.01-0.04 \mathrm{~Hz}$ ). However, the same patient often presents a mixture of breathing patterns, ranging from normal breathing (without cyclic modulation of ventilation) through mild $\mathrm{PB}$ to CSR patterns. Conventional spectral analysis assumes stationarity in the signal and is therefore unable to identify pattern changes. An approach which better accounts for such changes is the time-varying autoregressive (TVAR) model [10].

The aim of the present work is to characterize and study dynamic changes in the respiratory flow signal in CHF patients and healthy subjects. The characterization involves both spectral and temporal parameters extracted from the power spectrum of the respiratory flow envelope. The statistical distributions of these parameters account for the temporal evolution of the breathing pattern.

\section{ANALYZED DATA}

The respiratory flow signal was recorded from 35 healthy volunteers (12 males, 23 females, aged $26 \pm 7$ years), and 26 patients with CHF (19 males, 7 females, aged $65 \pm 9$ years) at Santa Creu i Sant Pau Hospital, Barcelona, Spain. All subjects were studied according to a protocol approved by the local ethics committee. The respiratory flow signals were registered using a pneumotachograph, consisting of a Datex-Ohmeda monitor with a Validyne Model MP45-1-871 Variable-Reluctance Transducer. The signals were recorded at $250 \mathrm{~Hz}$ sampling rate. According to clinical criteria, CHF patients were classified into two groups: 8 patients with periodic breathing pattern and 18 patients with non-periodic breathing pattern. For those with periodic breathing, 3 were classified as CSR and 5 as without apnea.

\section{Methodology}

\section{A. Signal preprocessing}

The respiratory flow signal is preprocessed with respect to artifact reduction. Firstly, outlier samples are removed which fall below the $1^{s t}$ percentile or above the $99^{t h}$ percentile. Next, short-duration spikes are removed by employing an auxiliary filtered signal, obtained as the original flow 
signal downsampled to $25 \mathrm{~Hz}$ and filtered by a median filter of order 11. Thus, samples for which the difference between the downsampled original signal and the auxiliary signal exceeds a threshold (here set to half the standard deviation of the signal) are replaced by the median value of neighboring samples. An interpolation method based on AR modeling is proposed in [11] which can handle missing samples. This method estimates the AR coefficients from the segment preceding the gap and then use the coefficients to forward predict the signal across the missing samples. The same process is repeated for the segment that follows the gap using instead backward signal extrapolation. The gap is then replaced by a cross-faded version of the two extrapolated predictions. Missing samples, defined as no respiratory activity, were observed and consequently replaced in two patients. Finally, considering that the respiratory frequency normally ranges from 0.2 to $0.4 \mathrm{~Hz}$, the signal is downsampled to $1 \mathrm{~Hz}$.

\section{B. Breathing pattern characterization}

The respiratory pattern characterization is based on the envelope of the respiratory flow signal, using the Hilbert transformation [12], [9].

1) Envelope extraction: The respiratory flow signal can be expressed by a deterministic lowpass signal modulated by a cosine with frequency $\omega_{m}$ and phase $\phi$ :

$$
s(n)=a(n) \cos \left(\omega_{m} n+\phi\right)
$$

where $s(n)$ is the respiratory flow signal and $a(n)$ the envelope of $s(n)$. The signal $s(n)$ can be expressed in the frequency domain as:

$$
S\left(e^{j \omega}\right)=\frac{1}{2}\left[A\left(e^{j\left(\omega-\omega_{m}-\phi\right)}\right)+A\left(e^{j\left(\omega+\omega_{m}+\phi\right)}\right)\right]
$$

where $S\left(e^{j \omega}\right)$ and $A\left(e^{j \omega}\right)$ are the discrete time Fourier transform of $s(n)$ and $a(n)$, respectively.

It is well-known that the envelope $a(n)$ is obtained by canceling out the negative frequencies and shifting the right side spectrum to origin [13]. In order to cancel the left side spectrum, the following function is defined:

$$
S_{A}\left(e^{j \omega}\right)=S\left(e^{j \omega}\right)+j H\left(e^{j \omega}\right) S\left(e^{j \omega}\right)=S\left(e^{j \omega}\right)+j \bar{S}\left(e^{j \omega}\right)
$$

where $H\left(e^{j \omega}\right)$ is the linear time-invariant filter that produces the Hilbert Transform, denoted $\bar{s}(n)$, the output of the Hilbert transform applied to the $s(n)$, that is a 90 shifted version of $s(n)$. Therefore, $s_{A}(n)$ represents a frequency shifted version of the envelope, and the positive valued envelope is obtained without any knowledge of $\omega_{m}$ or $\phi$.

$$
\begin{gathered}
s_{A}(n)=a(n) e^{j \omega_{m} n} \\
a(n)=\left|s_{A}(n)\right|=\sqrt{s^{2}(n)+\vec{s}^{2}(n) .}
\end{gathered}
$$

Since the frequency bandwidth of the flow envelope $a(n)$ is much lower than the original $s(n)$ signal, it is down-sampled to $0.1 \mathrm{~Hz}$. Fig. 1 shows as an example of
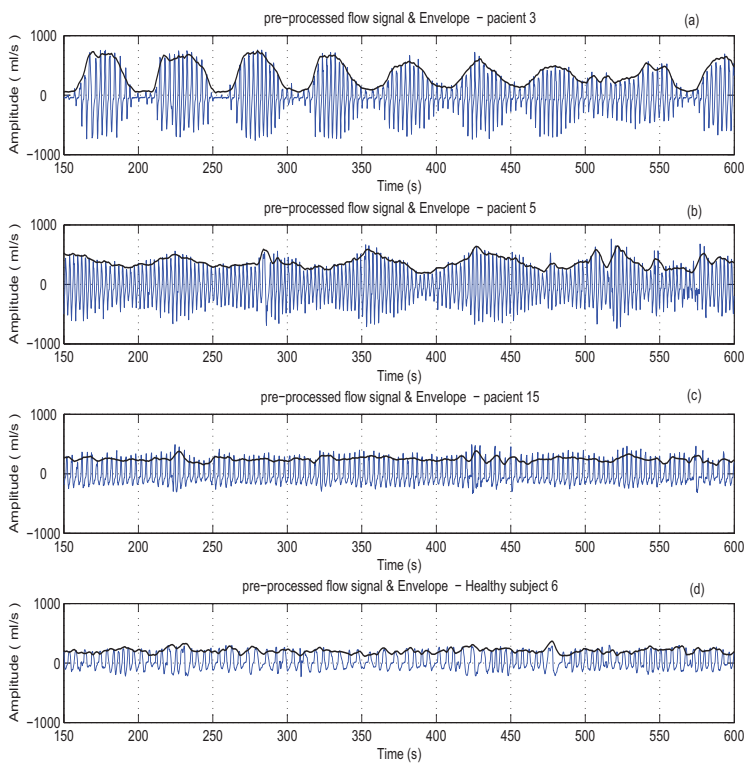

Fig. 1. Preprocessed flow signal and its envelope of (a) a CSR patient, (b) a patient with periodic breathing pattern, (c) a patient with non-periodic breathing pattern, and (d) a healthy subject.

the preprocessed respiratory flow signal and its envelope for a patient in each patient group of patients as well as for a healthy subject.

2) Time-varying spectral estimation: A time-varying AR algorithm may be used to model the respiratory envelope signal. Time-varying coefficients are determined through an adaptive method based on the recursive least squares (RLS) algorithm. It updates the previously evaluated sample on the basis of the prediction error, and weights it by means of a forgetting factor $\lambda \in(0,1]$. Small values of $\lambda$ imply high potential variability of the AR coefficients, while high values between (0.9-0.99) are usually more relevant in practice. The forgetting factor that minimize the least square error between the predicted envelope and the real one is selected from the recommended $\lambda$ range [10]. A value of 0.975 is obtained in average for CHF patients and 0.995 for healthy subjects. The envelope of the respiratory flow signal is with AR modeling obtained as:

$$
x(n)=-\sum_{k=1}^{p} a_{k}(n) x(n-k)+e(n)
$$

where $e(n)$ denotes zero-mean white noise with variance $\sigma^{2}$, $a_{k}(n)$ the AR coefficients, and $p$ the model order.

The selection of model order is a trade-off between the frequency resolution and the spurious peaks. The optimum model order is evaluated for each patient according to Rissanens minimum description length criterion. The mean of the optimum order of all CHF and healthy subjects $\left(4^{\text {th }}\right.$ order AR model) is selected as the most appropriate one. Power spectra are calculated at successive intervals from the 

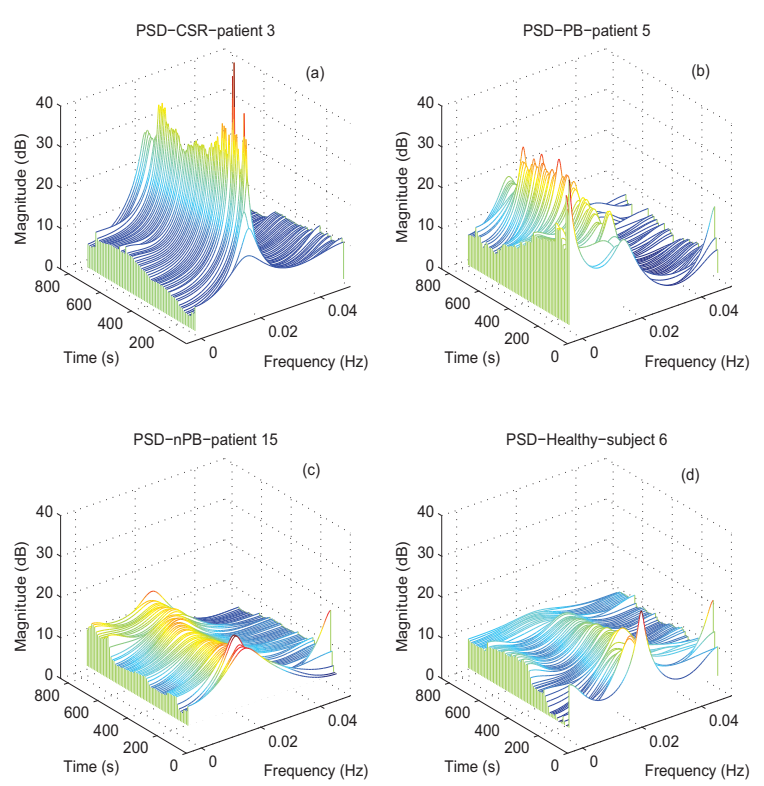

Fig. 2. Time-varying power spectrum of CHF patients and healthy subjects; (a) CSR patient, (b) PB patient, (c) nPB patient, (d) healthy subject.

estimated AR coefficients. Fig. 2 displays the time-varying power spectra of each $\mathrm{CHF}$ patient group ( $\mathrm{PB}, \mathrm{nPB}$ and CSR patient) and a healthy subject. The most striking feature in these diagrams is the temporal evolution of the modulation frequency peak.

3) Time-varying parameter extraction: Besides the AR coefficients and the two complex conjugate pole locations, various spectral parameters are extracted from the discriminant frequency band $(D B)$, which consist of the frequency interval $(0.02 \mathrm{~Hz})$ centered at the modulation frequency peak. The whole parameter set would characterize the behavior of the respiratory pattern each moment. However, to identify the most frequent pattern presented by each patient and healthy subject, the statistical distribution is evaluated for each time-varying parameter. Table 1 summarizes the different parameters and their statistics: mean $(\mathrm{m})$, standard deviation (s), interquartile range (i) and coefficient of variation (c).

TABLE I

PARAMETER DESCRIPTION AND STATISTICS

\begin{tabular}{ll}
$\mathbf{f p} ; m f p, s f p, i f p, c f p$ & Frequency peak \\
$\mathbf{P} ; m P, s P, i P, c P$ & Power of DB \\
$\mathbf{S} ; m S, s S, i S, c S$ & Slope from $f p$ to right DB end \\
$\mathbf{K} ; m K, s K, i K, c K$ & Kurtosis measure \\
$\mathbf{a}_{\mathbf{i}} ; m a_{i}, s a_{i}, i a_{i}, c a_{i}$ & AR coefficients $(i=1, \ldots, 4)$ \\
$\mathbf{r}_{\mathbf{i}} ; m r_{i}, s r_{i}, i r_{i}, c r_{i}$ & Poles magnitude $(i=1,2)$ \\
$\mathbf{p h}_{\mathbf{i}} ; m p h_{i}, s p h_{i}, i p h_{i}, c p h_{i}$ & Poles phase $(i=1,2)$ \\
\hline
\end{tabular}

\section{RESULTS}

Linear discriminant analysis is performed between 26 CHF patients and 35 healthy subjects in order to study the accuracy of the presented pattern characterization (Table 2). Firstly, CHF patients versus healthy subjects are classified, secondly nPB patients versus healthy subjects, and finally the internal $\mathrm{CHF}$ patient classification, $\mathrm{PB}$ versus $\mathrm{nPB}$. Through the statistics extracted from each parameter distribution, a feature selection is applied in order to select the most discriminant subset using leave-one-out crossvalidation. A high accuracy is obtained with only one parameter in all classifications. These results improve slightly with the addition of a second parameter.

TABLE II

Sensitivity (SN), SPECificity (SP), AND total ACCURACy, OBTAINED WITH THE BEST PARAMETERS FOR EACH CLASSIFICATION USING LEAVE-ONE-OUT CROSSVALIDATION.

\begin{tabular}{lllll}
\hline $\begin{array}{l}\text { Classi- } \\
\text { fications }\end{array}$ & $\begin{array}{l}\text { Best } \\
\text { Features }\end{array}$ & Sn & Sp & $\begin{array}{l}\text { Accu- } \\
\text { racy }\end{array}$ \\
\hline CHF vs. & $m r_{1}$ & $74,1 \%$ & $88,6 \%$ & $82,3 \%$ \\
Healthy & $m r_{1}+m P$ & $74,1 \%$ & $91,4 \%$ & $83,9 \%$ \\
\hline nPB-CHF & $m r_{1}$ & $73,7 \%$ & $82,9 \%$ & $79,6 \%$ \\
vs.Healthy & $m r_{1}+m P$ & $79,0 \%$ & $85,7 \%$ & $83,3 \%$ \\
\hline PB vs. & $c a_{1}$ & $75,0 \%$ & $89,5 \%$ & $85,2 \%$ \\
nPB (CHF) & $c a_{1}+m P$ & $75,0 \%$ & $94,7 \%$ & $88,9 \%$ \\
\hline
\end{tabular}

In spite of having present the accuracy of the most discriminant parameters, similar results have been observed with the other parameter's statistics: $m K, c K, c S l, m S$, and $c P$. Figs. 3, 4 and 5 correspond to the classification of CHF patients versus healthy subjects, nPB patients and healthy subjects and $\mathrm{nPB}$ versus $\mathrm{PB}$, into $\mathrm{CHF}$ patients, respectively.

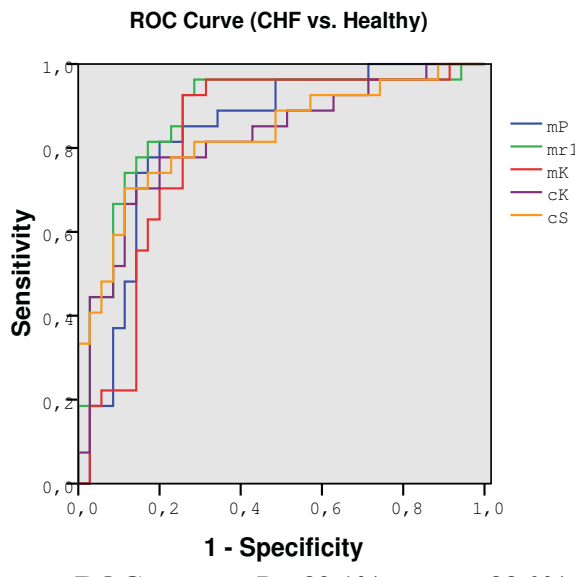

ROC area; $m P: 83,1 \%, m r_{1}: 88,0 \%$, $m K: 81,5 \% \quad c K: 81,8 \%, c S: 82,9 \%$

Fig. 3. ROC curves obtained with $m P, m r_{1}, m K, c K, c S$ and $m S$, classifying CHF patients versus healthy subjects. 


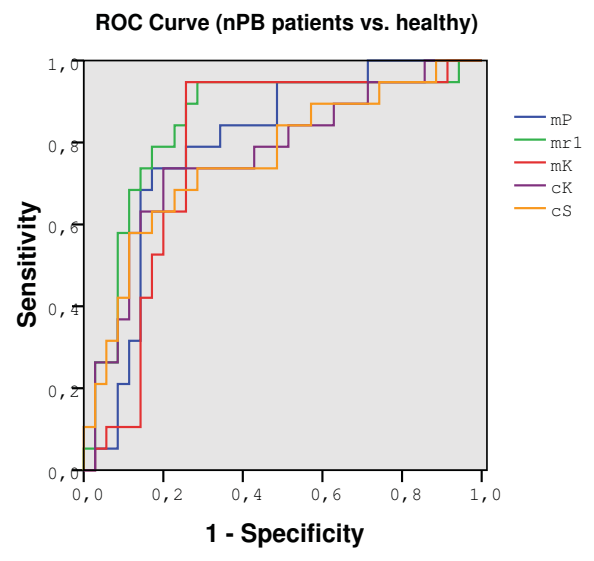

ROC area; $m P: 83,1 \%, m r_{1}: 88,0 \%$, $m K: 78,2 \% \quad c K: 76,5 \%, c S: 76,1 \%$

Fig. 4. ROC curves obtained with $m P, m r_{1}, m K, c K, c S$ and $m S$, classifying $\mathrm{nPB}$ patients versus healthy subjects.

ROC Curve (PB patients vs. nPB patients)

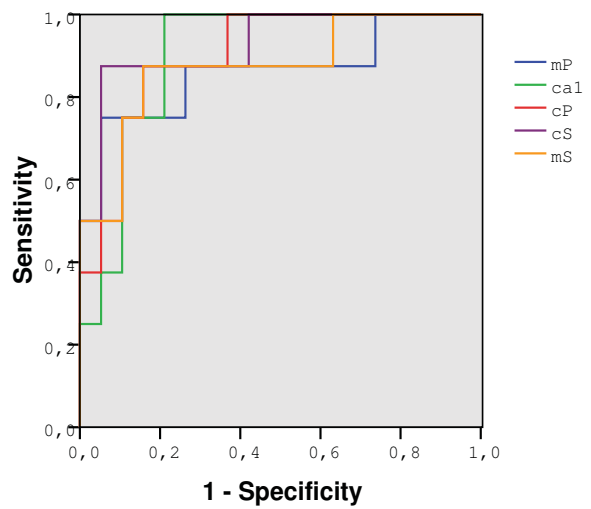

ROC area; $m P: 83,1 \%, m r_{1}: 88,0 \%$, $c P: 90,1 \%$ cS: $92,8 \%, m S: 87,5 \%$

Fig. 5. ROC curves obtained with $m P, m r_{1}, m K, c P, c S$ and $m S$, classifying $\mathrm{PB}$ versus $\mathrm{nPB}$ patients.

\section{CONCLUSIONS}

In this work, time-varying $\mathrm{AR}$ modeling is proposed for the characterization of respiratory flow signal patterns. The time-variant AR coefficients, pole localizations, and various spectral parameters can be used to study pattern's changes and classify CHF patients and healthy subjects. The statistic distribution is evaluated over each parameter to identify the predominant respiratory pattern. After feature selection, the percentage of the subjects correctly classified with the $m r_{1}$ (mean of the main pole magnitude) and the $m P$ (mean of the DB power) is $83,9 \%$ with $74,1 \%$ of sensitivity and $91.7 \%$ of specificity when classifying CHF patients versus healthy subjects, and $83,3 \%$, with $79,0 \%$ of sensitivity and $85,7 \%$ of specificity, when classifying $\mathrm{nPB}$ patients versus healthy subjects, instead. The parameters $m r_{1}$ and the $c a_{1}$ (coefficient of variation of the first $A R$ coefficient), selected as the most discriminant classifying $\mathrm{PB}$ and $\mathrm{nPB}$ into CHF patients, result in $88,9 \%$ of patients well classified with $75,0 \%$ of sensitivity and $94,7 \%$ of specificity. The ROCs validate the results obtained in all classifications with leave-one-out cross validation.

As a preliminary study, these results allow considering the time-varying modulation of flow envelope signal as a promising tool to characterize the temporal evolution of respiratory patterns. Further evaluation of the method's performance should be done on a larger set of flow signals.

\section{ACKNOWLEDGMENTS}

The authors would like to thank to Drs. S. Benito and A. Bayés-Genis and their collaborators of Santa Creu i Sant Pau Hospital, Barcelona, Spain, for their collaboration in the signal database acquisition.

\section{REFERENCES}

[1] G. Lorenzi-Filho, P. R. Genta, A. C. Figueiredo, and D. Inoue, "Cheyne-stokes respiration in patients with congestive heart failure: Causes and consequences," Clinics (Sao Paulo, Brazil), vol. 60, pp. 333-344, 2005.

[2] D. P. Francis, K. Wilson, L. C. Davies, A. J. Coats, and M. Piepoli, "Quantitative general theory for periodic breathing in chronic heart failure and its clinical implications," Circulation, vol. 102, pp. 2214 2221, 2000.

[3] G. D. Pinna, R. Maestri, A. Mortara, P. Johnson, T. Witkowski, P. Ponikowski, D. Andrews, S. Capomolla, M. La Rovere, and P. Sleight, "Nocturnal periodic breathing is an independent predictor of cardiac death and multiple hospital admissions in heart failure," in Proc. Comput. Cardiol. IEEE Press, 2006, pp. 837-840.

[4] G. D. Pinna, R. Maestri, A. Mortara, and M. T. La Rovere, "Cardiorespiratory interactions during periodic breathing in awake chronic heart failure patients," Am. J. Physiol. (Heart Circ. Physiol.), vol. 278, pp. H932-941, 2000.

[5] L. J. Findley, C. W. Zwillich, S. Ancoli-Israel, D. Kripke, G. Tisi, and K. M. Moser, "Cheyne-stokes breathing during sleep in patients with left ventricular heart failure," South. Med. J., vol. 78, pp. 11-15, 1985.

[6] J. P. Ribeiro, "Periodic breathing in heart failure: bridging the gap between the sleep laboratory and the exercise laboratory," Circulation, vol. 113, pp. 9-10, 2006.

[7] G. D. Pinna, R. Maestri, A. Mortara, M. T. L. Rovere, F. Fanfulla, and P. Sleight, "Periodic breathing in heart failure patients: testing the hypothesis of instability of the chemoreflex loop," J. Appl. Physiol., vol. 89, pp. 2147-2157, 2000.

[8] A. Garde, B. F. Giraldo, R. Jané, I. Diaz, S. Herrera, S. Benito, M. Domingo, and A. Bayes-Genis, "Characterization of periodic and non-periodic breathing pattern in chronic heart failure patients," in Proc. IEEE Conf. Eng. Med. Biol., 2008, pp. 3227-3230.

[9] A. Garde, B. Giraldo, R. Jané, I. Diaz, S. Herrera, S. Benito, M. Domingo, and A. Bayes-Genis, "Analysis of respiratory flow signals in chronic heart failure patients with periodic breathing," in Proc. IEEE Conf. Eng. Med. Biol., 2007, pp. 307-310.

[10] A. M. Bianchi, L. Mainardi, E. Petrucci, M. G. Signorini, M. Mainardi, and S. Cerutti, "Time-variant power spectrum analysis for the detection of transient episodes in HRV signal," IEEE Trans. Biomed. Eng., vol. 40, pp. 136-144, 1993.

[11] I. Kauppinen, J. Kauppinen, and P. Saarinen, "A method for long extrapolation of audio signals," J. Audio Eng. Soc., vol. 49, pp. 1167 $1180,2001$.

[12] L. Sörnmo and P. Laguna, Bioelectrical Signal Processing in Cardiac and Neurological Applications. Amsterdam: Elsevier (Academic Press), 2005.

[13] J. G. Proakis, Digital Communications, 4th ed. New Jersey: PrenticeHall, 2001. 